\title{
Diffusion of Cesium and lodine in Compressed IG-110 Graphite Compacts
}

L.M. Carter ${ }^{\mathrm{a}}$, J.D. Brockman ${ }^{\mathrm{b}}$, J.D. Robertson ${ }^{\mathrm{a}, \mathrm{b}}$, S.K. Loyalka ${ }^{\mathrm{c}}$

${ }^{a}$ Department of Chemistry, University of Missouri, 125 Chemistry Building, Columbia, MO 65211, United States

${ }^{b}$ University of Missouri Research Reactor Center, University of Missouri, 1513 Research Park Dr., Columbia, MO 65211, United

States

${ }^{c}$ Nuclear Science and Engineering Institute, University of Missouri, E2433 Lafferre Hall, Columbia, MO 65211, United States

*Corresponding author. Tel.: +1 5738848095

Email address: brockmanjd@missouri.edu

\section{Abbreviations}

HTGR: High Temperature Gas-Cooled Reactor

ICP-MS: Inductively Coupled Plasma-Mass Spectrometry

INAA: Instrumental Neutron Activation Analysis

FP: Fission Product

(C) 2016. This manuscript version is made available under the Elsevier user license http://www.elsevier.com/open-access/userlicense/1.0/ 
VHTR: Very High Temperature Reactor

BET: Brunauer-Emmett-Teller surface area

MURR $^{\circledR}$ : University of Missouri Research Reactor

\section{Abstract}

Nuclear graphite grade IG-110 is currently used in the High Temperature Engineering Test Reactor (HTTR) in Japan for certain permanent and replaceable core components, and is a material of interest in general. Therefore, transport parameters for fission products in this material are needed.

Measurement of diffusion through pressed compacts of IG-110 graphite is experimentally attractive because they are easy to prepare with homogeneous distributions of fission product surrogates. In this work, we measured diffusion coefficients for Cs and I in pressed compacts made from IG-110 powder in the 1079-1290K temperature range, and compared them to those obtained in as-received IG-110.

\section{Introduction}

The HTGR/VHTR utilizes graphite as a moderator, structural material, and barrier to fission product release. Diffusion coefficients for fission products in graphite are required for source term estimation, radiological surveillance, and reactor safety. Diffusion coefficients for fission products in graphite may be dependent on many variables including graphite type and properties (porosity, BET surface area, impurity concentration, and irradiation history), fission product identity, and concentration. The manufacturing method used to produce the graphite results in a characteristic microstructure described by the porosity, BET surface area, and pore size distribution. Irradiation causes structural defects in the graphite microstructure which include formation of vacancies, interstitials, and in-plane topological changes. 
The diffusion coefficients of fission products in graphite can be measured using a release method [1-7]. Advantages of the release method include non-destructive analysis and real time data collection. One challenge of the release method is in choosing the appropriate size of the graphite sample. A large sample may best represent the properties of bulk graphite but the time required to achieve a uniform distribution to satisfy the Booth model [4] using a technique that isopiestically infuses the element into the graphite $[1-3,5-6,8]$ can be prohibitive. Conversely, a uniform distribution of an infused element can be achieved quickly with a small graphite sample but the sample may not represent bulk graphite due to variability of the pore structure. A second important limitation of the release method is the difficulty of infusing low-volatility elements into graphite samples. For example, the challenge of loading a graphite sphere with Sr has limited the usefulness of the release method for measuring the $\mathrm{Sr}$ diffusion coefficient. These two challenges may be overcome by measuring diffusion in graphite compacts made from graphite powder instead of bulk graphite. Surprisingly, it has been reported that Sr diffusion coefficients obtained from diffusion measurements in some bulk graphites are similar to those obtained from pressed graphite compacts made from powdered graphite of the same type [9]. This suggests that the mechanism of Sr diffusion is relatively insensitive to differences in the microstructure of pressed graphite pellets and manufactured graphite. An additional advantage of compacted graphite is that it is relatively simple to load multiple elements to fabricate a surrogate fission spectrum.

The objective of the present work is to compare the measured diffusion coefficients of Cs and I in pressed graphite pellets and bulk graphite. If the diffusion in compacted graphite and bulk graphite is similar, then compacted graphite may be used to approximate the diffusion of $\mathrm{Ag}$, $\mathrm{Sr}$, and other important fission products in bulk graphite when isopiestic infusion is not feasible. Differences between the graphite microstructure of the pressed pellets and the manufactured graphite should result in different Cs and I diffusion coefficients. The underlying mechanisms of I transport in graphite are not 
described well by Fickian kinetics [5]. However, for the purposes of fuel performance modeling codes, I diffusion is handled using Fickian kinetics with an effective diffusion coefficient that encompasses pore diffusion, grain diffusion, trapping, and other complex transport mechanisms [10]. In this work, we compare the diffusion of Cs and I in compacted IG-110 graphite to our previsouly published work on CS and I diffusion in bulk IG-110 graphite [1,5].

The diffusion of Cs and I in graphite compacts in measured using the release method, where the source of Cs and I in graphite compacts is Csl. As noted above, we investigated graphite compacts because it is desirable to start with a graphite sample that contains a uniform distribution of diffusant to simplify the boundary conditions for the diffusion equation.

\section{Theory}

For our purposes here, the diffusion of a species in a sample is described by the equations:

$$
\begin{aligned}
& \frac{\partial C(\mathbf{r}, t)}{\partial t}=D \nabla^{2} C(\mathbf{r}, t) \\
& C(\mathbf{r}, 0)=C_{0} \\
& C\left(\mathbf{r}_{s}, t\right)=0
\end{aligned}
$$

where, $C(\mathbf{r}, t)$ is the concentration of the fission product $\left(\mathrm{g} / \mathrm{m}^{3}\right), C\left(\mathbf{r}_{\mathrm{s}}, t\right)$ is the concentration of the fission product at the surface of the sample, $D$ is the diffusion coefficient $\left(\mathrm{m}^{2} / \mathrm{s}\right), \mathbf{r}$ is the distance vector $(\mathrm{m})$, and $t$ is the time $(\mathrm{s}) . C_{0}$ is a constant; the initial uniform concentration $\left(\mathrm{g} / \mathrm{m}^{3}\right)$. We also assume that $C(\mathbf{r}, t)$ is finite everywhere. The diffusion coefficient $D$ is determined by comparing the

experimental fractional release rate, $f(t)$ or the cumulative fractional release $F(t)$ with the corresponding theoretical results. The release rates are defined as: 


$$
f(t)=\frac{1}{V C_{0}} \int\left(-D \frac{\partial C\left(\mathbf{r}_{s}, t\right)}{\partial n}\right) d S
$$

And,

$$
F(t)=\int_{0}^{t} f\left(t^{\prime}\right) d t^{\prime}
$$

Where $V$ is the volume of the sample, and $\mathbf{n}$ is a unit normal vector at $\mathbf{r}_{s}$ a point on the surface and is directed outwards from the body.

For a finite-cylindrical sample of radius $R$ and length $h$, one gets:

$$
F(t)=\sum_{m=1}^{M} \sum_{n=1}^{N} b_{m . n}\left(1-\exp \left[-\lambda_{m . n} D t\right]\right)
$$

Where:

$$
b_{m, n}=\frac{32}{m_{0}^{2} n^{2} \pi^{2}}, \text { if } \mathrm{n} \text { is odd; } 0, \text { otherwise. }
$$

Also,

$$
\lambda_{m . n}=\left(\frac{m_{0}}{R}\right)^{2}+\left(\frac{n \pi}{h}\right)^{2}
$$

Here $m_{0}$ is the $m$ th root of the Bessel Function of order zero, $J_{0}$. The series converges slowly as $M$ and $N$ go to infinity (we have later used $M=100$ and $N=100$, and explored up to $M=200$ and $N$ 
=200). Note that $\sum_{m=1}^{M} \sum_{n=1}^{N} b_{m . n} \rightarrow 1$, as $M, N \rightarrow \infty$. For fast computations, we have separated the terms and summations in Equation (4) as:

$$
\begin{aligned}
F(t) & =\frac{32}{\pi^{2}}\left(\sum_{m=1}^{M} \frac{1}{m_{0}^{2}}\right)\left(\sum_{n=1,3,5, . .}^{N} \frac{1}{n^{2}}\right)-\frac{32}{\pi^{2}}\left(\sum_{m=1}^{M} \frac{1}{m_{0}^{2}} \exp \left[-\left(\frac{m_{0}}{R}\right)^{2} D t\right]\right)\left(\sum_{n=1,3,5, . .}^{N} \frac{1}{n^{2}} \exp \left[-\left(\frac{n \pi}{h}\right)^{2} D t\right]\right) \\
& \approx 1-\frac{32}{\pi^{2}}\left(\sum_{m=1}^{M} \frac{1}{m_{0}^{2}} \exp \left[-\left(\frac{m_{0}}{R}\right)^{2} D t\right]\right)\left(\sum_{n=1,3,5, . .}^{N} \frac{1}{n^{2}} \exp \left[-\left(\frac{n \pi}{h}\right)^{2} D t\right]\right)
\end{aligned}
$$

And this works as well as the series solution for a sphere. Still, since the series converges slowly, it is useful to consider a short time approximate solution also. For a sphere of radius $R_{s}$ the short time solution is particularly simple:

$$
F_{\text {short }}(t)=6 \sqrt{\frac{\tau}{\pi}}-3 \sqrt{\tau}
$$

Where the dimensionless quantity $\tau$ is the reduced time, viz:

$$
\tau=\frac{D t}{R_{S}^{2}}
$$

Equation (8) is accurate to an experiment time that results in a fractional release up to about $90 \%$ of the diffusant (i.e. for values of $\tau$ less than approximately 0.2 ) [11]. With appropriate normalizations, Equation (8) is also suitable for describing diffusion from non-spherical samples at short time scales. The surface flux of diffusant, at short times, emanates from a layer close to the sample surface; therefore, this approximation is valid when the concentration at the center of the sample has not yet depleted substantially. Thus, for a sample of an arbitrary shape, the radius of a surface area equivalent sphere is defined as: 


$$
R_{\text {eqsphere }}=\left(\frac{S_{\text {sample }}}{4 \pi}\right)^{1 / 2}
$$

Setting:

$$
V C_{0} F(t) \approx V_{\text {eqsphere }} C_{0} F_{\text {eqsphere }}(t)
$$

Where $V_{\text {eqsphere }}$ is the volume of the surface area equivalent sphere of radius $R_{\text {eqsphere }}$ and assuming the initial concentration to be the same in the sample as well as the equivalent sphere. Thus we have:

$$
F(t) \approx \hat{\alpha} F_{\text {eqsphere }}(t)
$$

with

$$
\hat{\alpha}=V_{\text {eqsphere }} / V=\frac{4 \pi}{3}\left(\frac{S}{4 \pi}\right)^{3 / 2} / V
$$

a geometrical factor. For the finite cylinder that we are considering, we have,

$$
R_{\text {eqsphere }}=\left(\frac{1}{2} R(h+R)\right)^{1 / 2}
$$

and,

$$
\hat{\alpha}=\frac{\sqrt{2}}{3} \frac{R}{h}\left(1+\frac{h}{R}\right)^{3 / 2}
$$

We have used Equation (7) to fit the experimental data directly for the entire time period. For the short time period, we have used, Equation (12), viz:

$$
\hat{\alpha}\left(6 \sqrt{\frac{D t}{\pi R_{\text {eqsphere }}^{2}}}-3 \frac{D t}{R_{\text {eqsphere }}^{2}}\right)=F(t)
$$


The variable and non-homogeneous structure of graphite significantly complicates the theory, as the transport of diffusant becomes dependent on the many variables (trapping, porosity, etc.) mentioned previously. As such, it is commonplace to approximate graphite as a homogeneous medium and describe the diffusant transport in terms of an effective diffusivity $D=D_{\text {eff, }}$ and this is the approach taken here. We discuss the data and the results for the diffusion coefficient $D$ in the next sections.

\section{Experimental}

\subsection{Instrumentation}

A Perkin Elmer NexION ${ }^{\circledR} 300 X$ scanning quadrupole ICP-MS system was used for quantification of the release rates of Cs and I from the IG-110 samples. The ICP-MS utilized a Glass Expansion ${ }^{\circledR}$ dual port quartz spray chamber for simultaneous introduction of the sample and standard. A nebulizer continuously introduced a $1.00 \mathrm{ppb}$ indium standard in $2 \% \mathrm{HNO}_{3(\mathrm{aq})}$ at $200 \mu \mathrm{L} / \mathrm{min}$ into one port of the spray chamber in order to correct for instrument variation during the measurements.

Cs and I released from the samples was transported to other port of the spray chamber using a helium jet which contained a carbon aerosol. A Palas ${ }^{\circledR}$ GFG-1000 spark discharge aerosol generator operating at 0.7 bar $\mathrm{He}$ and $50 \mathrm{~Hz}$ spark frequency was used to produce the aerosol. A custom SiC cell was housed in a Thermo ${ }^{\circledR}$ Lindberg-Blue HTF- $55322 \mathrm{C}$ tube furnace; the SiC cell contained the samples as well as inlet and outlet tubes for the clean aerosol and Cs/I laden product aerosol, respectively. The outlet tube of the SiC cell was connected to the spray chamber of the ICP-MS using stainless steel

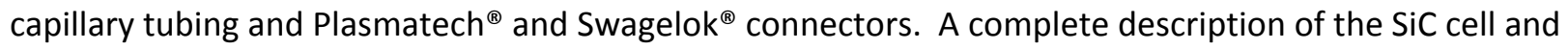
calibration procedures is given in Refs [1-3].

\subsection{Materials and Methods}

\subsubsection{Sample preparation}


As-received IG-110 stock material was milled into powder and sized using mesh sieves. Particles which aggregated between the $20 \mu \mathrm{m}$ and $38 \mu \mathrm{m}$ mesh screens were used for fabrication of compacts. A $0.1 \mathrm{mg} / \mathrm{g}$ solution of cesium iodide in $1.5 \mathrm{M}$ aqueous ammonia was prepared. We added $5 \mathrm{~mL}$ of the solution to $5 \mathrm{~g}$ of the IG-110 powder and the resulting slurry was mixed thoroughly and dried in air. The $\mathrm{Cs} / \mathrm{I}$ laden sample was subsequently heated to approximately $100^{\circ} \mathrm{C}$ under vacuum to drive off remaining moisture. Cylindrical compacts were fabricated from the Cs/I laden powder using a Reflex Analytical ${ }^{\circledR} 1 / 8 "$ non-evacuable pellet die set (model \#2804). The powder was compressed under 2.22 kN for 30 seconds. Sample dimensions were measured using a micrometer. The solution used to dope the IG-110 powder contained Cs and I initially in the $\mathrm{Cs}^{+}{ }_{\text {(aq) }}$ and $\mathrm{I}_{(\text {(aq) }}$ forms, respectively. The chemical forms of Cs and I present within the graphite after fabrication of the pellets were not determined experimentally.

\subsubsection{Initial Cs and I content analysis}

The Cs and I content in the compacts was measured using standard comparator neutron activation analysis at the $\mathrm{MURR}^{\circledR}$ facility. Cs comparator standards were prepared from a certified solution of $\mathrm{CsNO}_{3}$ in $2 \%$ aqueous $\mathrm{HNO}_{3}$ purchased from High Purity Standards ${ }^{\circledR}$. I standards were prepared from a certified solution of I in $0.01 \% \mathrm{KOH}$ from Fisher Scientific ${ }^{\circledast}$. The sample and standards were irradiated in the row 2 pneumatic tube irradiation position for ten seconds at a thermal flux of $5.0 \times 10^{13} \mathrm{n} / \mathrm{cm}^{2} / \mathrm{s}$ to produce ${ }^{134 \mathrm{~m}} \mathrm{Cs}$ and ${ }^{128} \mathrm{I}$ via the reactions ${ }^{133} \mathrm{Cs}(\mathrm{n}, \mathrm{\gamma})^{134 \mathrm{~m}} \mathrm{Cs}$ and ${ }^{127} \mathrm{I}(\mathrm{n}, \mathrm{\gamma})^{128} \mathrm{I}$,

respectively. The $127.5 \mathrm{keV}$ gamma ray from decay of ${ }^{134 \mathrm{~m}} \mathrm{Cs}$ and the $442.9 \mathrm{keV}$ gamma ray from decay of ${ }^{128}$ I were measured using a high purity Ge detector.

The mass of each of the compacts and a control compact were measured using a MettlerToledo $^{\circledR}$ analytical balance. The concentrations of Cs and I (g Cs/g graphite or g I/g graphite) in the 
control sample were used to compute the initial mass of Cs and I within the other compacts. These concentrations were determined to be $200 \pm 10 \mu \mathrm{g}$ Cs/g and $300 \pm 15 \mu \mathrm{g} \mathrm{I/g}$.

\subsubsection{Diffusion measurements}

Cs and I released from the compact samples were transported to a Perkin Elmer NexION ${ }^{\circledR} 300 \mathrm{X}$ ICP-MS using a carbon aerosol He-jet system and SiC diffusion cell assembly described in detail in Refs. [1-3] and Section 3.1. Release from the graphite spheres was measured isothermally for approximately 1.5 hours at temperatures in the range of $1079 \mathrm{~K}$ to $1290 \mathrm{~K}$.

\subsubsection{Final Cs and I content analysis}

After release measurements, the Cs and I remaining in the samples was quantified again using INAA methods similar to those described in section 3.2.2.

\section{Results}

The initial distribution of Cs and I in the samples is assumed to be uniform and the samples are assumed to be uniformly and isotropically compacted. The concentrations of Cs and I in the IG-110 powder used to fabricate the compacts were $200 \pm 10 \mu \mathrm{g}$ Cs/g IG-110 and 300 $\pm 15 \mu \mathrm{g} \mathrm{I/g} \mathrm{IG-110,}$ respectively. The dimensions for each sample, relevant parameters, and diffusion coefficients calculated via Equations (7) and (16) are given in Table 1 below and Figures 1 and 2 below:

Table 1. Measured diffusion coefficients and related experimental parameters for cesium and iodine diffusion in pressed IG-110 compacts.

\begin{tabular}{lllllll}
\hline $\begin{array}{l}\text { Temperature } \\
(\mathrm{K})\end{array}$ & $\begin{array}{l}\text { Radius } \\
(\mathrm{cm})\end{array}$ & $\begin{array}{l}\text { Length } \\
(\mathbf{c m})\end{array}$ & $\begin{array}{l}\boldsymbol{D}_{c s}\left(\mathrm{~m}^{2} \mathrm{~s}^{-1}\right) \\
\text { Series } \\
\text { solution }\end{array}$ & $\begin{array}{l}\boldsymbol{D}_{c s}\left(\mathrm{~m}^{2} \mathrm{~s}^{-1}\right) \\
\text { Equivalent } \\
\text { sphere } \\
\text { approximation }\end{array}$ & $\begin{array}{l}\boldsymbol{D}_{l}\left(\mathrm{~m}^{2} \mathrm{~s}^{-1}\right) \\
\text { Series } \\
\text { solution }\end{array}$ & $\begin{array}{l}\boldsymbol{D}_{l}\left(\mathrm{~m}^{2} \mathrm{~s}^{-1}\right) \\
\text { Equivalent } \\
\text { sphere } \\
\text { approximation }\end{array}$ \\
\hline 1079 & 0.159 & 0.384 & $5.6 \times 10^{-12}$ & $5.4 \times 10^{-12}$ & $2.9 \times 10^{-10}$ & $3.6 \times 10^{-10}$ \\
1140 & 0.159 & 0.191 & $4.4 \times 10^{-12}$ & $4.2 \times 10^{-12}$ & $1.2 \times 10^{-10}$ & $1.2 \times 10^{-10}$ \\
1188 & 0.159 & 0.292 & $3.2 \times 10^{-11}$ & $3.2 \times 10^{-11}$ & $2.1 \times 10^{-10}$ & $1.9 \times 10^{-10}$
\end{tabular}




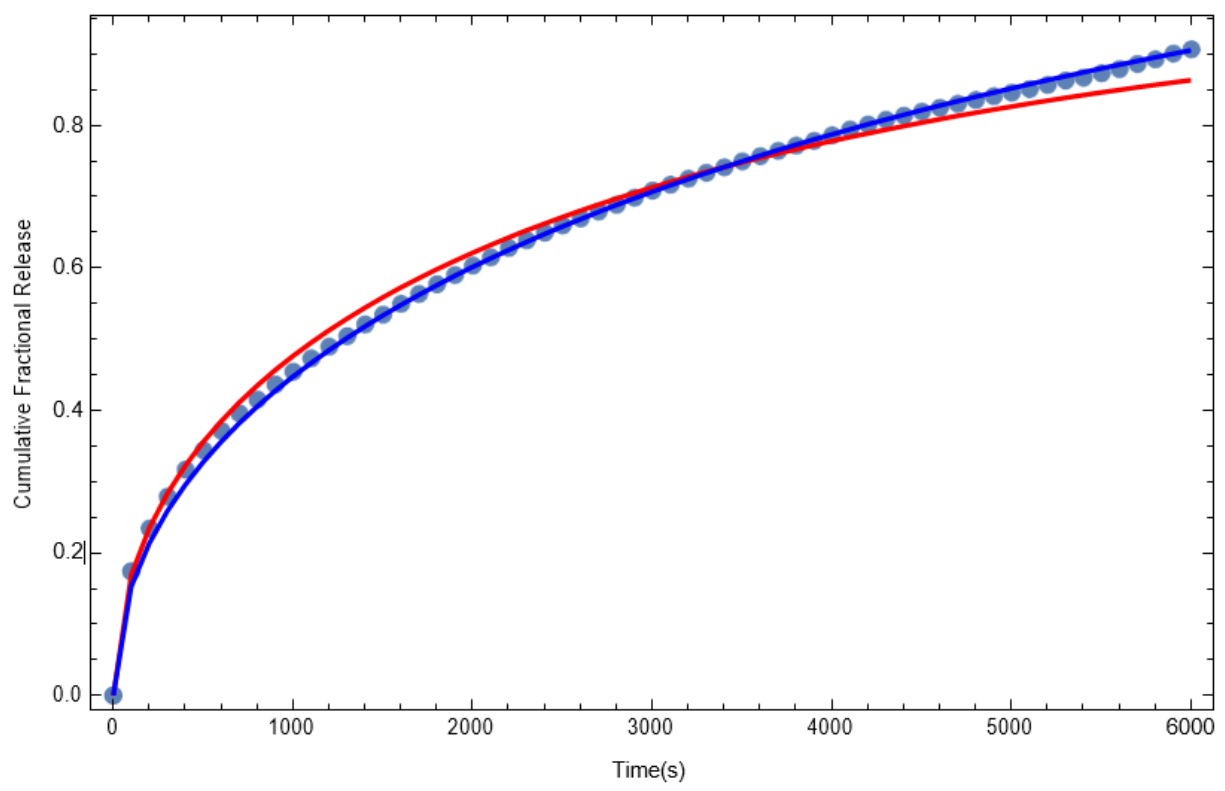

Figure 1: Fractional release of Cs from IG-110 cylindrical compact at $T=1290 \mathrm{~K}$. The blue circles are the measured data, the red lines are the series fit $\left(D_{C s}=7.2 \times 10^{-11} \mathrm{~m}^{2} / \mathrm{s}\right)$ and the blue lines are the fit of equivalent sphere approximation $\left(D_{c s}=5.4 \times 10^{-11} \mathrm{~m}^{2} / \mathrm{s}\right)$. 


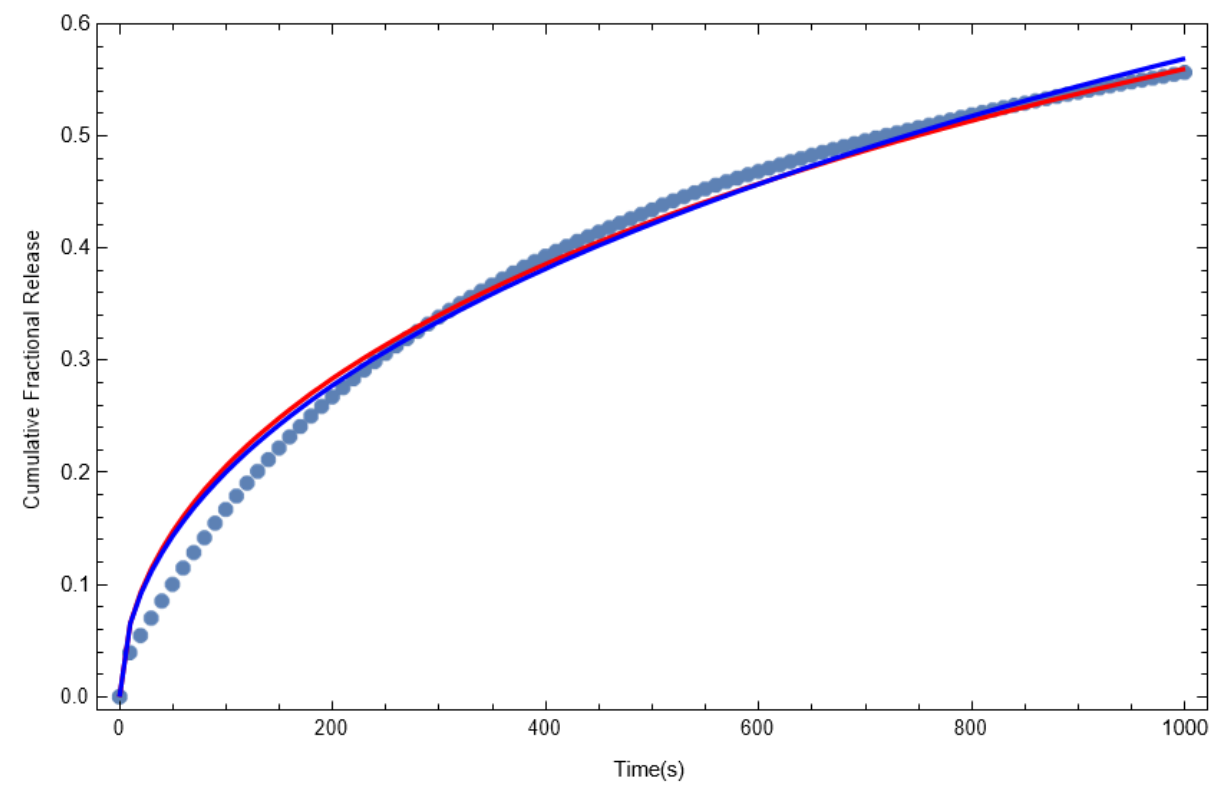

Figure 2: Fractional release of I from IG-110 cylindrical compact at $T=1290 \mathrm{~K}$. The blue circles are the measured data, the red lines are the series fit $\left(D_{l}=1.1 \times 10^{-10} \mathrm{~m}^{2} / \mathrm{s}\right)$ and the blue lines are the fit of equivalent sphere approximation $\left(D_{l}=9.4 \times 10^{-11} \mathrm{~m}^{2} / \mathrm{s}\right)$.

Diffusion coefficients were calculated via least squares fit of the data with the series solution for the fractional release (Equation 7) to $M=200$ and $N=200$, as well as the short time equivalent sphere approximation (Equation 15) for comparison. In the equivalent sphere approximation fits, only the data points corresponding to fractional releases of less than 0.5 from the cylindrical samples was considered, as at $F(t)>0.5(\tau>0.03)$ the equivalent sphere approximation begins to diverge significantly from the true solution for the sample geometry tested here. Differences in the diffusion coefficients calculated from the fits of Equations (7) and (16) are therefore largely due to a longer measurement interval being considered in the series fits, rather than divergence of the theoretical expressions for $F(t)$.

The temperature dependence of the diffusion coefficient is classically given by the following Arrhenius equation: 


$$
D=D_{0} \exp \left(\frac{-E_{a}}{R T}\right)
$$

where $E_{a}$ is the activation energy for diffusion, $R$ is the universal gas constant, and $T$ is the absolute temperature. We report the pre-exponential and activation parameters for Cs diffusion in Table 2 below; additionally in Table 2 we have included results from previous investigations of Cs and I diffusion in bulk IG-110 graphite for comparison:

Table 2. Pre-exponential and activation parameters for Cs diffusion and I diffusion in the present work (pressed compact) and previous investigations [1,5]:

\begin{tabular}{lllllll}
\hline Material & $\begin{array}{l}\text { Infusion } \\
\text { source }\end{array}$ & Diffusant & $D_{0}\left(\mathrm{~m}^{2} / \mathrm{s}\right)$ & $\pm \Delta / n D_{0}$ & $E_{a}(\mathrm{~J} / \mathrm{mol})$ & $\pm \Delta E_{a}(\mathrm{~J} / \mathrm{mol})$ \\
\hline $\begin{array}{l}\text { IG-110 Pressed } \\
\text { Compact }\end{array}$ & $\mathrm{Csl}$ & $\mathrm{Cs}$ & $1.3 \times 10^{-4}$ & 4.27 & $1.6 \times 10^{5}$ & $4.2 \times 10^{4}$ \\
\hline IG-110 Pressed & $\mathrm{CsI}$ & $\mathrm{I}$ & $1.3 \times 10^{-12}$ & 2.4 & $-4.7 \times 10^{4}$ & $2.4 \times 10^{4}$ \\
Compact & & & & & & \\
IG-110 Infused [1] & $\mathrm{CsNO}_{3}$ & $\mathrm{CS}$ & $1.0 \times 10^{-7}$ & 2.90 & $1.1 \times 10^{5}$ & $2.8 \times 10^{4}$ \\
IG-110 infused [5] & $\mathrm{CsI}$ & $I$ & $6 \times 10^{-12}$ & 1.1 & $-3.0 \times 10^{4}$ & $1.0 \times 10^{4}$ \\
IG-110 Infused [5] & $I_{2}$ & $I$ & $4 \times 10^{-10}$ & 0.96 & $1.1 \times 10^{4}$ & $8.0 \times 10^{3}$ \\
\hline
\end{tabular}

\section{Discussion}

We previously measured diffusion coefficients of Cs and I in IG-110 spheres infused with Cs and IG-110 spheres infused with I. The diffusion coefficients for Cs in the pressed IG-110 powder compacts tested here are approximately an order of magnitude larger than in Cs-infused spheres machined from the as-received bulk material (see Figure 3). This implies that Cs diffusion rates are largely dependent on one or a combination of the following factors: a) the porosity and pore structure, b) the presence of I, and c) the initial concentration of Cs. In contrast, the results for I diffusion in the pressed compacts were not observed to be significantly different than those obtained for I diffusion in bulk IG-110 infused using either CsI or molecular $\mathrm{I}_{2}$. Additionally, the results for the I diffusivity in the IG-110 compacts show a decreasing trend with increasing temperature, reflected in the negative value for the activation energy 
$E_{\mathrm{a}}$. The $P$-value for the slope of $\ln D_{1}$ vs $1 / T(P=0.14)$ for the compact measurements suggests the slope is not significantly different from zero. This slope is expected to be small due to the generally weak binding of I to graphite (physisorption) observed in previous work $[5,12]$. We have investigated this previously [5] and concluded that I diffusion is not well-described by simple Fickian kinetics. Higher temperatures may promote the dissociation of molecular $\mathrm{I}_{2}$ to form the radical $\mathrm{I} \cdot$ which interacts more strongly with the graphite (chemisorption) than physisorbed molecular $I_{2}$. Diffusion coefficients from several previous analyses of Cs and I diffusion are presented in Figure 3 and Figure 4 below, for comparison with the results in the present work:

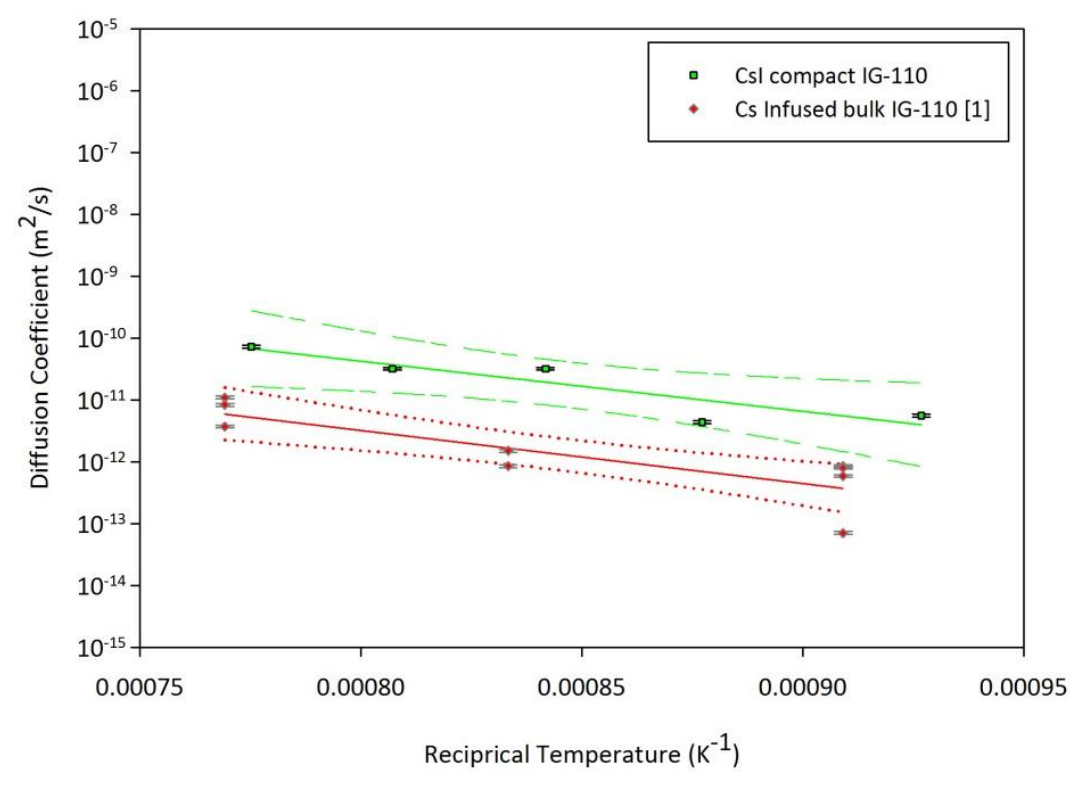

Figure 3: A comparison of the diffusion coefficients of $\mathrm{Cs}$ in bulk IG-110 loaded as $\mathrm{CsNO}_{3}$ (diamonds, Refs [1]) and Cs in IG-110 compacts loaded as CsI (squares, present work). The data is plotted with the linear regression line and $95 \%$ confidence interval. 


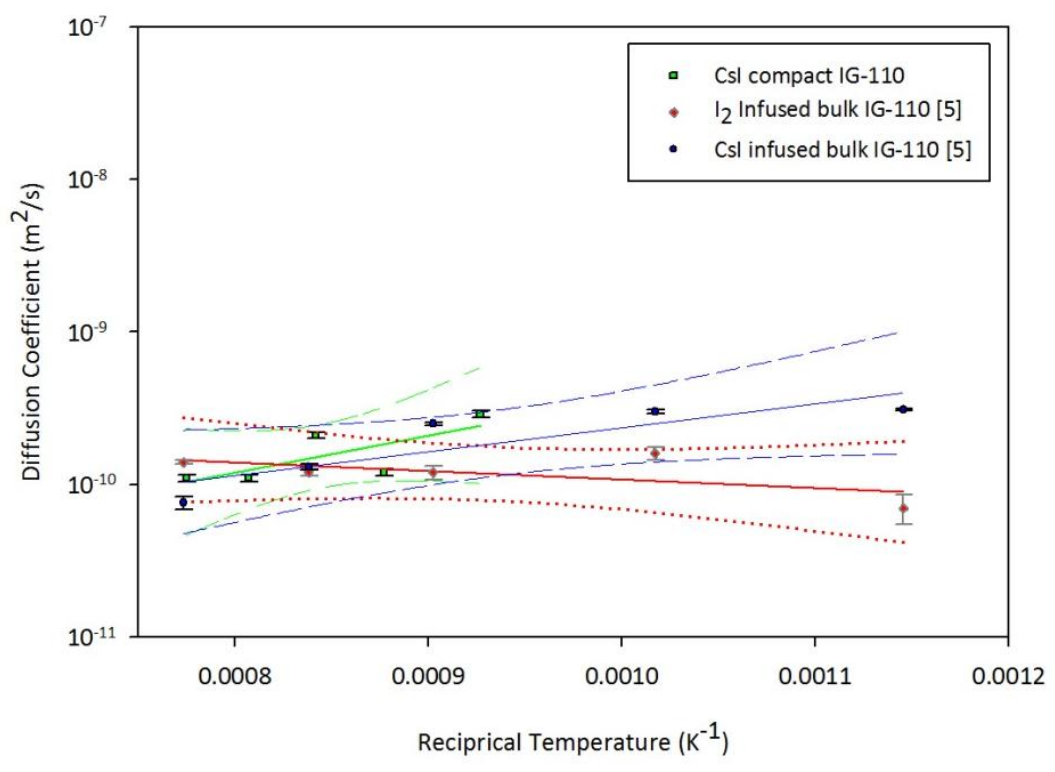

Figure 4: A comparison of the diffusion coefficients of I in bulk IG-110 loaded as CsI (diamonds, Refs [5]), $\mathrm{I}_{2}$ and IG-110 compact loaded with CsI (squares, present work). The data is plotted with the linear regression line and $95 \%$ confidence interval.

Figure 5 shows SEM images of the cylindrical compact material and the as-received IG-110 material. Differences in the morphology of the as-received and pressed compact IG-110 image are not as evident as expected. The samples appear similar at the micron scale and below. We note that in both cases, the infused surrogate fission products, I and Cs, are expected to be present largely on the pore surfaces, grain boundaries, and in the binder components. In contrast, in-pile I and Cs produced via fission of uranium contamination in the graphite is expected to initially reside within graphite grains to a larger extent, due to recoil of these nuclides into the graphite lattice. Cs and I released from failed fuel particles is likely finer characterized by the present models. 

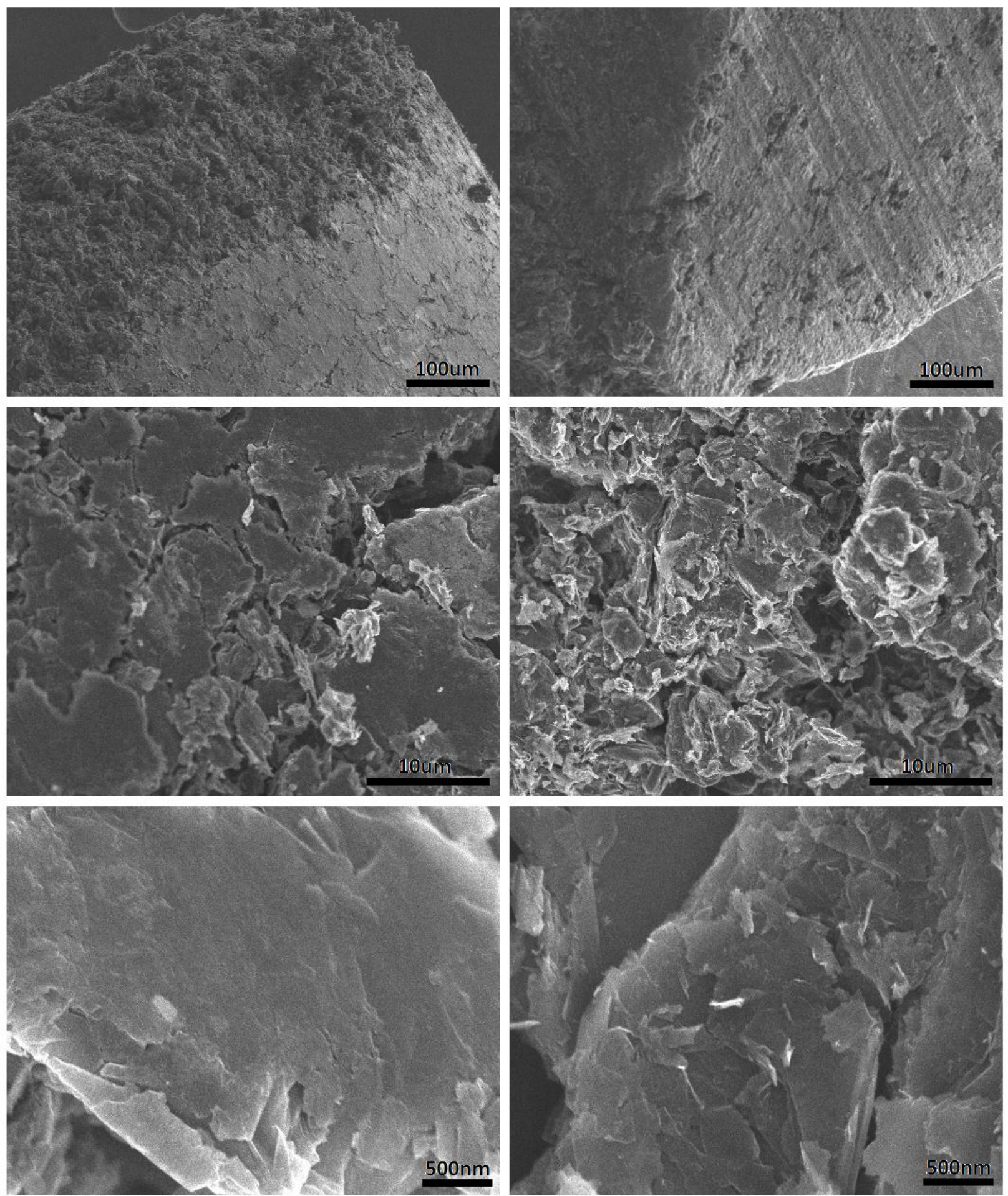

Figure 5: Uniaxially compressed IG-110 powder (left) and as-received IG-110 (right) imaged using a Hitachi ${ }^{\circledR}$ S-4700 field emission scanning electron microscope. 
The predominant chemical forms of diffusing Cs and I in HTGRs remain the subject of debate. Idealized calculations have revealed the most stable form for I at low oxygen potentials is actually Csl; however, previous laboratory and reactor cooling circuit analyses have indicated the I behavior is better characterized by atomic $\mathrm{I}$ or molecular $\mathrm{I}_{2}$ [12]. In the present work, we have observed that the diffusion coefficients and release rates for I remain substantially higher than those for Cs for temperatures up to 1290K. If indeed the predominant form of diffusing Cs and I were Csl, one would expect the diffusion coefficients for Cs and I to be the same, which was not observed in the temperature range tested.

In contrast to previous work for $\mathrm{Sr}$, the diffusion coefficients for $\mathrm{Cs}$ in the pressed compacts of IG-110 were larger than those obtained from spheres of bulk IG-110 infused with Cs. The diffusivity results for I from the pressed compacts were similar to our previous work with spheres of bulk IG-110 graphite [5]. The significant difference in the results for the Cs diffusivity between the pressed compacts and bulk IG-110 suggest that Cs transport in graphite is more sensitive than I to the graphite pore structure and morphology, and further, that compacted IG-110 is an inadequate proxy for the bulk material. This is not surprising because milling the bulk material into powder fundamentally changes the pore size distribution, degree of interconnected porosity, density, and may introduce contamination through machining processes. In the present case, however, the Cs diffusivity difference appears nominal considering manufacturing inhomogeneities in IG-110 have been shown to impart over an order of magnitude of variation in measured diffusion coefficients in the bulk material [13]. Still, we intend to investigate alternate methods for infusion of surrogate fission products into graphite which more accurately represent the in-pile material.

\section{Conclusion}


We have measured diffusion coefficients of Cs and I in uniaxially compressed IG-110 graphite using the release method. Release rates of Cs and I were simultaneously measured via an online ICP-MS coupled with a carbon aerosol helium gas-jet system. This appears to be the first real-time measurement of multicomponent diffusion in graphite. The diffusion of Cs in compacted IG-110 graphite is not representative of Cs diffusion in bulk IG-110 graphite. Interestingly, we did not observe a significant difference between I diffusion in compacted IG-110 graphite and I diffusion in bulk IG-110 graphite.

\section{Acknowledgements}

This research has been supported by the U.S. Department of Energy Nuclear Energy University Program (NEUP-2982). The authors express gratitude to Saint Gobain-Hexaloy ${ }^{\circledR}$ and the MURR ${ }^{\circledR}$ Science Instrument Shop for mechanical support.

\section{References}

[1] L.M. Carter, J.D. Brockman, S.K. Loyalka, J.D. Robertson. Measurement of cesium diffusion coefficients in graphite IG-110. J. Nucl. Mater. 460 (2015) 30-36.

[2] L.M. Carter, J.D. Brockman, J.D. Robertson, S. K. Loyalka. ICP-MS Measurement of cesium diffusion coefficients in NBG-18 graphite. J. Nucl. Mater. 466 (2015) 402-408.

[3] L.M. Carter, J.D. Brockman, J.D. Robertson, S. K. Loyalka. Calibration of a system for measurements of diffusion coefficients of fission products in HTGR/VHTR core materials. J. Radioanal and Nucl. Ch. 307 (2016) 1771-1775.

[4] A.H. Booth, A method of calculating fission gas diffusion from UO2 fuel and its application to the X-2f loop test. Atomic Energy of Canada Ltd., 1957. Technical report AECL-496. 
[5] L.M. Carter, J.D. Brockman, J.D. Robertson, S. K. Loyalka. ICP-MS Measurement of iodine diffusion in IG-110 graphite for HTGR/VHTR. J. Nuc. Mater. 473 (2016) 218-222.

[6] H.J. Leyers. Cesium release out of graphitic matrix A3 in flowing helium. E. Hoinkis (Ed.), Transport of Fission Products in Matrix and Graphite. HMI-B372. Proceedings of a Colloquium held at Hahn-Meitner Institut, Berlin, 1981 (published, June 1983), p. 103.

[7] E. Hoinkis. The determination of diffusion coefficients of cesium and silver by the release method in as received, oxidized and neutron irradiated graphitic matrix. E. Hoinkis (Ed.), Transport of Fission Products in Matrix and Graphite. HMI-B372. Proceedings of a Colloquium held at Hahn-Meitner Institut, Berlin, 1981 (published, June 1983), p. 77.

[8] K. Hilpert. Sorption of strontium by graphitic materials. Berichte der Bunsengesellschaft für Physikalische Chemie. Volume 89, Issue 1, p. 43-48, (1985).

[9] F. J. Sandalls, M.R. Walford, Laboratory determinations of strontium diffusion coefficients in graphite. J. Nucl. Mater. 62 (1976) 265-72.

[10] K. Verfondern, J. Cao, T. Liu, H.J. Allelein. Conclusions from V\&V studies on the German codes PANAMA and FRESCO for HTGR performance and fission product release. Nucl. Eng. Des. 271 (2014) 8491.

[11] D. R. Olander, Fundamental Aspects of Nuclear Fuel Elements. Energy Research and Development Administration. 1976.

[12] O. K. Tallent, R. P. Wichner, R. L. Towns, T. T. Godsey. Analysis of iodine and cesium chemical forms evolved from graphite surfaces at temperatures from 452 to $1400^{\circ} \mathrm{C}$. 1985 . HTGR-85-069 ORNL/TM9218, Oak Ridge National Laboratory, Tennessee, US. 
[13] K. Hayashi, K. Fukuda, Diffusion coefficients of cesium in un-irradiated graphite and comparison with those obtained from in-pile experiments. J. Nuc. Mater. 168 (1989) 328-336. 